INNOVACIÓN

\title{
Estrategia para la enseñanza de las relaciones internacionales en la carrera de Derecho en Cuba
}

\author{
Estratégia para o ensino de relações internacionais na carreira do Direito em Cuba
}

Strategy for teaching international relations in the Law university studies in Cuba

\author{
Adoración Carballo Moya \\ Universidad Pinar del Río, Cuba \\ Lisett Daymaris Páez Cuba \\ Universidad de Pinar del Río, Cuba
}

RESUMEN La relación intrínseca entre la epistemología jurídica y la didáctica del derecho constituye el punto de partida para abordar el proceso de enseñanza-aprendizaje del derecho en las universidades. A su vez, el contexto internacional y el desarrollo de las relaciones multifacéticas actuales aclaman nuevos enfoques inter, multi y transdisciplinarios con respecto al iusinternacionalismo. El objetivo de este trabajo está dirigido a fundamentar una estrategia pedagógica para la enseñanza de las relaciones internacionales en la carrera de Derecho en Cuba, de manera que tribute a la formación integral del jurista. Se analizará la interconexión entre dos ciencias: el derecho y las relaciones internacionales, con particular incidencia en el abordaje del derecho internacional. Además, se hará una revisión de planes de estudios tanto a nivel nacional como internacional, los cuales sugieren la previsión curricular de competencias profesionales genéricas y específicas. La propuesta aborda una estrategia pedagógica concebida para lograr el rediseño curricular del derecho internacional como disciplina científica, la transversalidad de la enseñanza de las relaciones internacionales en la carrera de Derecho y la inclusión de un sistema de actividades extracurriculares que potencien los juegos de roles profesionales en el estudiante de derecho.

PALABRAS CLAVE Competencias, derecho, didáctica, pedagogía, relaciones internacionales. 
RESUMO A intrínseca relação entre a epistemologia jurídica e a Didática do Direito constitui o ponto de partida para abordar o processo de ensino-aprendizagem do Direito nas Universidades. Por sua vez, o contexto internacional e o desenvolvimento das relações multifacetadas atuais aclamam as novas abordagens inter, multi e transdisciplinares do direito internacional. O objetivo deste trabalho é estabelecer uma estratégia pedagógica para o ensino das Relações Internacionais na carreira do Direito em Cuba, de modo que contribua para a formação integral do jurista. É analisada a interligação entre duas ciências: Direito e Relações Internacionais, com particular incidência na abordagem do Direito Internacional. É realizada uma revisão dos currículos no nível nacional e internacional, que sugerem a previsão curricular de competências profissionais genéricas e específicas. A proposta aborda uma estratégia pedagógica concebida para o redesenho curricular do Direito Internacional como disciplina científica, a transversalidade do ensino das Relações Internacionais na carreira do Direito e a inclusão de um sistema de atividades extracurriculares que promovam a atuação profissional na o estudante de direito.

PALAVRAS-CHAVE Competências, direito, didática, pedagogia, relações internacionais.

ABSTRACT The intrinsic relationship between the legal epistemology and the Didactics of Law constitutes the starting point to approach the teaching-learning process of Law in the Universities. In turn, the international context and the development of current multifaceted relations, need of new inter, multi and transdisciplinary approaches about the ius-internationalism. The objective of this work is aimed at founding a pedagogical strategy for teaching international relations in the university studies of Law in Cuba, so that it contributes to the comprehensive training of the jurist. The interconnection between two sciences is analyzed: Law and International Relations, with particular emphasis on the approach of International Law. A review of foreign and national curricula is carried out, which suggests the curricular forecast of generic and specific professional competences. The proposal addresses a pedagogical strategy conceived to achieve the curricular redesign of International Law as a scientific discipline, the transversality of the teaching of international relations in the Law university studies and the inclusion of a system of extracurricular activities that enhance professional role-plays in the Law student.

KEYWORDS Competences, law, didactics, pedagogy, international relations.

\section{Introducción}

Los procesos de enseñanza aprendizaje del derecho y de las relaciones internacionales - considerando la autonomía de ambas ciencias - invocan, en primer lugar y desde una perspectiva diacrónica, a los estudios jurídicos. Era precisamente desde el derecho, la diplomacia y la historia, que se intentaba conocer la dinámica mundial, 
sin que se materializara todavía una disciplina propia dedicada a las relaciones internacionales. Fue en la Universidad de Bolonia en 1088, vanguardista en los estudios sociales, donde se fundó la carrera de Derecho. No obstante, no fue hasta el siglo XX que emergieron las relaciones internacionales, cuyo origen como ciencia y método data de 1919 en la Universidad de Gales (actualmente Universidad de Aberystwyth) gracias a la iniciativa del filántropo David Davies, quien se planteó el objetivo de favorecer y potenciar un marco académico que abordase los problemas relacionados con el derecho, la política, la ética y la economía desde una perspectiva global (Izquierdo, 2019: 3).

Es a partir del fenómeno de la globalización que se entiende la imbricación de las relaciones internacionales con la ciencia jurídica y la retroalimentación que deben tener los estudios de ambas ciencias. En ese sentido, Calduch (2018: 9) enarbola el supuesto teórico de que la globalización surge como resultado de la combinación de un nuevo medio de comunicación: internet, que operando a través de unos nuevos sistemas de telecomunicaciones móviles se ha extendido vertiginosamente por una sociedad internacional, que ya tenía un alcance mundial en las relaciones estratégicas, económicas, de transporte y de comunicaciones, pero que todavía estaba fragmentada política y culturalmente. Asimismo, el autor se cuestiona cómo afectará este nuevo proceso globalizador al desarrollo del orden común internacional si de una parte está potenciando la conciencia de pertenencia individual a una comunidad universal de comunicación por encima de las barreras estatales, étnicas, religiosas o lingüísticas, pero de otra parte se está generando una economía virtual, en la que la producción y el consumo de bienes y servicios, al igual que las transacciones financieras, están deslocalizadas y, en gran medida, desreguladas, lo que liberaliza los flujos comerciales y financieros que hasta ahora eran regulados y controlados por los Estados. No obstante, con la sociedad virtual también están surgiendo nuevas amenazas y actividades delictivas que afectan al núcleo mismo de la seguridad de las personas, los grupos sociales y los Estados, provocando inestabilidad política, desequilibrios económicos y enfrentamientos culturales sobrevenidos que todavía son escasamente prevenidos, regulados y perseguidos (Calduch, 2018: 9).

Ante los desafíos globales y locales, con una nueva configuración suscitada por el contexto de la covid-19, donde en un primer momento el virus se localizaba en China, en la ciudad de Wuhan, deviniendo luego una problemática mundial, se aboga por un modelo que permita lidiar con la complejidad a través de la ciencia de la sostenibilidad. Al respecto, el catedrático cubano Núñez (2020:5) alega que esta «debe ser consciente de los riesgos y la provisionalidad de sus propuestas, y deberá generar vínculos efectivos con la política, con el propósito de crear una mutua comprensión entre los académicos y quienes toman decisiones».

Siendo esta la perspectiva de la ciencia cubana del siglo XXI, y a tono con el numeral cuarto de los Objetivos del Desarrollo Sostenible dirigido a una educación 
de calidad, la formación en relaciones internacionales opera como alternativa viable para la consecución de este fin. En la carrera de Derecho, específicamente en pregrado, esta formación amerita un redimensionamiento de los diseños curriculares. Como acertadamente se ha alegado, con respecto al ámbito jurídico «los procesos de perfeccionamiento y/o modificación del plan de estudios persiguen fortalecer la formación integral de los estudiantes» (Delgado, 2017: 7).

El redimensionamiento de los contenidos tradicionalmente impartidos conlleva a la inserción de una metodología que, "de manera innovadora y creativa», solucione las problemáticas del contexto. En ese sentido, la asunción de un diseño curricular por competencias es un enclave fundamental en la investigación educativa, y en Cuba constituye una novedad debido a la formulación de currículos por objetivos.

En el propio país caribeño, se estudian las carreras de Derecho y Relaciones Internacionales, la primera en 16 universidades del territorio nacional y la segunda en el Instituto Superior de Relaciones Internacionales (ISRI) de La Habana. Ambas tienen en común el estudio de derecho internacional. Sin embargo, esta conexidad es limitada, ya que requieren de un derecho del influjo de las relaciones internacionales que trascienda el iusinternacionalismo.

En ese sentido, el objetivo de este trabajo se centra en fundamentar una estrategia pedagógica para la enseñanza de las relaciones internacionales en la carrera de Derecho en Cuba, de manera que tribute a la formación integral del jurista. Su consecución se logra a través del uso de métodos como la revisión documental, la observación científica y la entrevista grupal, empleados en esta investigación que resulta ser de tipo empírico cualitativa.

Para la consecución de los fines investigativos, se asumen los aportes a las relaciones internacionales de Truyol (1973), Velázquez (2006), Rodríguez (2017), García (2017), Calduch (2018) e Izquierdo (2019). En el campo de la pedagogía, se sustenta la apoyatura teórica en autores como Álvarez (1999), Valle (2007), Villalpando (2009) y, específicamente en materia de didáctica del derecho, los aportes de Magendzo y Toledo (2015), Delgado (2017), Hernández (2018), Ramallo (2020) y Del Mastro (2020). Asimismo, de los estudios sobre la didáctica de las relaciones internacionales en la carrera de Derecho se constataron las contribuciones de Páez, Silva y Trujillo (2020). Todos estos constituyen bases teóricas que complementan los resultados de la matriz DAFO y sustentan la estrategia pedagógica propuesta como resultado principal de la investigación.

\section{La ciencia jurídica y las relaciones internacionales: Puntos de conexión trascendentes a la didáctica}

La relación intrínseca entre la epistemología de una ciencia y su didáctica resulta insoslayable, criterio validado por Álvarez (1999: 23), quien advirtiera la necesidad de emplear, consecuentemente, «el método de la ciencia como método fundamental 
de enseñanza y aprendizaje». Esta relación de carácter interdisciplinar se pondera en este estudio, toda vez que el abordaje de la doctrina de las relaciones internacionales resulta esencial para la comprensión cabal de la didáctica de las relaciones internaciones, así como el estudio de la ciencia jurídica es requisito sine qua non para perfeccionar la enseñanza del derecho.

Sobre la base de este primer planteamiento, los aislamientos teóricos entre la ciencia jurídica y la teoría de las relaciones internacionales resultan objetables, de manera que la doctrina iusinternacionalista coincide en no verlas por separado, sino en ponderar la relación de interconexión y complementariedad entre ambas. De hecho, los antecedentes de las relaciones internacionales se encuentran en el derecho romano (a través del ius gentes) y en la diplomacia (heredada de Grecia). Así, se gesta una epistemología en torno a la internacionalidad, ${ }^{1}$ que trasciende a la actualidad.

En cuanto al derecho, la jurista cubana Prieto (2019) lo define como un fenómeno social complejo, que designa un comportamiento social condicionado por elementos socioeconómicos, políticos, ideológicos, culturales y con un contenido axiológico. A su vez, el derecho es catalogado por Narváez (2019: 6) como concepto plurívoco, cuyo objeto debe rebasar lo meramente normativo, razón por la cual coincidimos en identificar «la relación jurídica» como objeto de estudio de la ciencia del derecho.

Con respecto a las relaciones internacionales, para Zimern (Sipaco, 2014: 3) estas constituyen «un estudio científico del mundo contemporáneo, en la búsqueda de un contenido nuevo, pero aprovechando todo lo que sea aprovechable de las ciencias sociales». Por su parte, Calduch define que «el objeto material de esta disciplina es la realidad social internacional (sociedad internacional), que solo parcialmente coincide con la realidad interestatal, pero que resulta más amplia y compleja al incluir individuos y grupos distintos del Estado» (Calduch, 2013: 13).

Para Truyol (1973: 12), las relaciones internacionales son definidas como:

Aquellas relaciones entre individuos y colectividades humanas que en su génesis y su eficacia no se agotan en el seno de una comunidad diferenciada y considerada como un todo, que fundamentalmente (pero no exclusivamente) es la comunidad política o Estado, sino que trasciende sus límites.

Es por esto que la propia denominación convierte a las relaciones internacionales en una ciencia omnímoda, y esta no es una etiqueta que se acepta a priori, ya que la evolución que ha tenido hasta ganar autonomía corrobora que los estudios internacionales son abarcadores, aglutinando un cúmulo de conocimientos imprescindibles para entender la dinámica mundial.

1. Aunque aludir al término «internacionalidad» es una ficción debido a la inexistencia entonces del Estado nación, pero homologado a la interacción territorial que desarrollaron estas civilizaciones con los restantes pueblos. 


\section{¿Estudios jurídicos internacionales o estudios internacionales del jurista en formación de pregrado?}

Si se tiene en cuenta el criterio validado por Calduch (2013: 13), relativo a que «las relaciones internacionales constituyen una disciplina científica diferenciada y autónoma del resto de las ciencias sociales», se puede entender por qué el derecho es, con respecto a dicha disciplina, una ciencia auxiliar general, toda vez que aporta conceptos, métodos o explicaciones que afectan al conjunto de conocimientos desarrollados por la ciencia de las relaciones internacionales. En esta categoría se encuentran, además, la historia, la economía, la ciencia política y la sociología (Calduch, 2011: 8).

Por su parte, el derecho considera las relaciones internacionales como los estudios de posgrado a la manera de una supuesta especialización del jurista que, dentro de sus esferas de actuación, puede convertirse en cónsul o devenir un analista internacional a través de programas de diplomado, maestría o doctorado.

En cuanto al área del pregrado de derecho, la mayor proximidad para comprender la realidad internacional es el derecho internacional, lo cual reduce el amplio espectro de las relaciones internacionales. Esto se puede entender desde dos puntos de vista. De una parte, como refiere Calduch (2011: 3), Max Huber considera que con el derecho internacional se puede apreciar la internacionalidad configurada en su sentido más estricto, el jurídico, ya que «son las relaciones entre los Estados mismos». De otra parte, Rodríguez (2017) sitúa al derecho internacional como uno de los procesos reguladores del sistema internacional, por lo que no es el exclusivo, existiendo otros como el equilibrio de fuerzas y las organizaciones internacionales.

Por lo tanto, el estudiante de derecho se apropia de conocimientos de la asignatura internacionalista y, a través de ella, percibe y puede explicar los fenómenos que tienen lugar a nivel mundial. No obstante, como argumenta Salvioli (2002: 2):

Hay una percepción de poca eficacia del derecho internacional, por los continuos ejemplos de violaciones al mismo que se observan desde el conocimiento transmitido a través de agentes no formales de educación (por ejemplo, los medios masivos de difusión), y desde no pocos círculos jurídicos.

El propio autor refiere que el derecho internacional, como acertadamente se queja Remiro Brotóns (2002: 2), «parece condenado a justificar, del big bang al apocalipsis, su fundamentación y validez jurídica».

También se pretende avanzar hacia la universalidad de las normas internacionales de manera que se consolide una categoría superior para así traducir la voluntad colectiva de la comunidad internacional. Requiere de la imperatividad y la automática ilicitud de los actos y de la responsabilidad ante cualquier violación. Sin embargo, la aplicación efectiva de estas normas está limitada y esto ocurre como resultado de la resistencia a su cumplimiento, que sostienen en la práctica numerosos y poderosos 
Estados, lo cual es imprescindible para la cabal aplicación de estas normas de ius cogens, además de que existen otras dificultades de la comunidad internacional para imponer su imperatividad (Calduch, 2018: 16).

Como acertadamente refiere Calduch (2018: 16):

La tensión dialéctica entre las dinámicas política y jurídica obliga a considerar el derecho internacional de nuestros días como un derecho propio de una etapa de transición entre sociedades internacionales, eludiendo así las interpretaciones simplistas realizadas desde una visión unilateral y exclusiva.

Por lo tanto, la toma de decisiones y otras condicionantes políticas son las que transgreden el efectivo cumplimiento del derecho internacional, por lo que estas eventualidades condicionan un reto pedagógico encaminado a lograr la inserción de la ideología política de las relaciones internacionales en la carrera de derecho, asumida en el presente estudio desde la transdisciplinariedad.

La transversalidad de las relaciones internacionales al derecho se entiende a partir de que este es un fenómeno social en expansión producto de la globalización. Al respecto, el autor Waldo Villalpando (2009: 16) menciona que:

Hasta hace algunos años, el derecho internacional quedaba confinado al campo estrecho de las relaciones internacionales interestatales estudiado en el llamado derecho internacional público y la casuística individual que alimentaba el derecho internacional privado. Hoy, en cambio, las relaciones entre los Estados se han intensificado, de modo que ya no se puede prescindir del estudio de normas interregionales, por ejemplo, las de la Comunidad Europea o el Mercosur.

Para el propio Villalpando (2009: 16):

Las relaciones jurídicas empresariales también se han globalizado y es esencial considerar los contratos de comercio a la luz de distintos derechos nacionales y usos comerciales. En el tradicional derecho penal, se ha abierto ya el camino de una nueva disciplina, el derecho internacional penal, que da obligatoriedad de sanción a comportamientos delictivos considerados ahora crímenes internacionales, con una actividad que supera a la tradicional cooperación jurídica entre Estados. El derecho está trascendiendo fronteras al estudiar la regulación jurídica de las comunicaciones, la prevención de la salud, la protección medioambiental, las convenciones internacionales de uso y explotación del mar.

La vocación internacionalista del estudiante, a través de la impartición de temas de política internacional, condiciona una serie de competencias, donde, conociendo del pragmatismo en la dinámica mundial, desarrolla un pensamiento crítico que le permite conocer los riesgos y amenazas que desde el sistema internacional persuaden el derecho. Además, en menor escala se genera el «internacionalescepticismo», como 
tendencia negativa asociada a la apreciación del derecho internacional.

Siguiendo esta misma línea, Salvioli (2002: 2) argumenta en cuanto al derecho internacional que:

La asignatura es vista - a priori- por los alumnos y alumnas como muy compleja, y sin utilidad visible para el desarrollo profesional medio, salvo para quienes pretenden seguir la carrera diplomática, o que experimentan una vocación docente en torno a la materia, y desean formarse como profesoras o profesores de derecho internacional público [la cursiva es nuestra].

Esta percepción desalentadora, que suelen tener los estudiantes en torno al derecho internacional, amerita un rediseño didáctico y epistémico, ambos pertinentes para la comprensión cabal del sistema internacional. A tono con lo anterior, se ha acuñado que el derecho internacional:

Requiere más que antes de nuevos enfoques teóricos, modelos y esquemas didácticos que procuren su construcción académica, básicamente entre profesores y alumnos, para proyectar su aprendizaje y aplicación en el convulso mundo que hoy vivimos con sus múltiples y cambiantes escenarios» (Estrada y Reyes, 2015: 910).

En este sentido, según Velázquez (2006), existen dos enfoques o tendencias en la enseñanza del derecho internacional: el tradicional y el moderno. El primero de ellos adquiere una perspectiva formalista, con fórmulas anglosajonas, principalmente europea. Sin embargo, el enfoque moderno se sostiene sobre una perspectiva analíticocrítica, con una metodología científica más dinámica y abierta. Es esta segunda tendencia la que se asume como base de sustentación para el diseño de una estrategia que, de manera sistémica e integrada, tribute a perfeccionar el proceso formativo abordado.

Hasta lo analizado hasta aquí, se entiende que la carrera de Derecho dota al estudiante de las herramientas necesarias para realizar estudios jurídicos internacionales, frente a lo cual es menester que desarrolle la competencia genérica, consistente en la realización de estudios internacionales que trasciendan el iusinternacionalismo.

Luego de analizar que efectivamente el derecho internacional es una parte imprescindible de las relaciones internacionales, se acuña el criterio de Velázquez al respecto, ya que «solo un ignorante del derecho internacional puede pretender «desjuridificar» las relaciones internacionales» (Velázquez, 2006). Se impone, por ende, el análisis de la otra parcela de esta ciencia, que trasciende a la formación del jurista.

Debido a que las expectativas de la investigación sobrepasan el mero estudio del derecho internacional, y siendo las relaciones internacionales una disciplina holística, se infiere que incorporar su estudio en el diseño curricular del derecho fomentaría la formación integral del jurista, dotándole de una visión más general de los fenómenos, los conceptos y la dinámica internacional que atraviesan al derecho, configurán- 
dose un puente ineludible entre estas dos ciencias.

\section{El rol de las relaciones internacionales en la formación de pregrado en la carrera de Derecho}

En la carrera de Derecho, un indicador de que el estudiante no suele analizar el fenómeno jurídico desde las relaciones internacionales es su concepción de la prolongación de los tiempos de paz, siendo muy poco estudiado el terreno de los riesgos y amenazas que afronta el derecho en tiempos de guerra. En particular, las situaciones excepcionales atañen a los estudios constitucionales, pero inclusive las propuestas de modificación constitucional y del resto de las normas se sustentan desde la inclusión de nuevos derechos, procedimientos y principios que, en su mayoría, operan ante una estabilidad en la vida cotidiana. ¿Acaso el derecho solo debe preocuparse por un normal desenvolvimiento de las relaciones acaecidas en tiempos normales? ¿El jurista está preparado para preocuparse también por las tensiones que puedan afectar en el orden estatal el statu quo?

Si bien el derecho mayoritariamente está diseñado para tiempos de paz, sucede que las relaciones internacionales trascienden al escenario bélico o de conflictos. Tanto es así que otras ciencias consideran a las guerras, las revoluciones, el espionaje, las conquistas, la injerencia extranjera y el poder nacional como situaciones excepcionales, dentro de ellas la sociología y el derecho, siendo aquellos los objetos normales de estudio para las relaciones internacionales.

Sobre su carácter abarcador, el propio Velázquez (2006: 836) afirma que «las relaciones internacionales son una ciencia heurística y analítica, esto es, de una gran utilidad para explicar, por ejemplo, el proceso legislativo y normativo jurídico internacional, sus limitaciones y alcances». De ahí su relación entre derecho y relaciones internacionales en un sentido amplio.

Siendo insuficientes las competencias que se adquieren a través del derecho internacional para la formación en relaciones internacionales del estudiante de derecho, se impone el perfeccionamiento de este proceso. A nivel internacional, se evidencia que, en algunas carreras como Periodismo de la Universidad de Valladolid (2015), existe una asignatura llamada Relaciones internacionales del mundo contemporáneo.

Autores como Farfán (2019), Solanes (2018) y Bellver (2019) han abordado el tema sobre cómo perfeccionar la formación del jurista desde distintas perspectivas, lo cual, en el ámbito de la investigación educativa, es un enclave central en el progreso del proceso de enseñanza aprendizaje. Desde la retórica, «la formación del novel profesionista consistiría en aprender un lenguaje y, a la vez, apropiarse de un acto profesional total o forma de pensar el mundo, de sentir y de actuar en él de manera profesional» (Farfán, 2019: 10).

Desde los estudios iusfilosóficos, «si lo que se pretende es formar juristas capaces 
de razonar, argumentar, reflexionar, en definitiva, de pensar, entonces el modelo de formación no puede ser estrictamente técnico y, por tanto, la importancia de las materias filosófico-jurídicas será evidente» (Solanes, 2018:7). Sigue plenamente vigente la distinción hecha por Álvarez, hace casi dos siglos, y que recuerda Pérez Luño, en la que se diferenciaba entre «leguleyos» como meros repetidores de leyes, «jurisperitos» como técnicos en controversia legal, «rábulas» como aquellos que se quedan en el estudio crudo de la ley y, finalmente, «jurisconsultos» como quienes saben aplicar las leyes «erudita y juiciosamente». En este último punto, se puede ubicar al «buen» jurista. Así mantiene Solanes (2018: 7), quien al citar a Pérez Luño alega que:

La organización didáctica de los estudios jurídicos que persiga formar auténticos juristas requerirá impartir disciplinas de carácter iusfilosófico, en cambio, si se quiere producir leguleyos, jurisperitos y rábulas es posible prescindir de las consideraciones crítico-valorativas que la perspectiva filosófica de los problemas jurídicos aporta.

Otros autores, como Bellver (2019), se han referido a una formación común para los distintos currículos a través de la educación en derechos humanos, donde se ha planteado por el autor que se debería instituir una materia de derechos humanos en todos los grados universitarios. Además, y no es secundario, debe desarrollarse, a criterio de otros juristas, un «programa de estudios que aborde las áreas de derecho de lo general a lo particular, de lo simple a lo complejo, y señalando en cada especialidad la metodología que la caracteriza» (Villalpando, 2009: 24).

Luego de lo analizado, se coincide en que no es menos cierto que esa búsqueda de una formación integral del jurista tenga los anteriores sustentos, pero muchos de ellos están inmersos en la imbricación de las relaciones internacionales como estrategia para contribuir a dicha formación.

Por otra parte, la retórica está implícita en uno de los modos de actuación del analista internacional, lo que puede homologarse desde la perspectiva válida para el derecho. No se pretende saturar de exigencias al jurista en formación, pero si en algo coinciden las ciencias en cuestión es en el desarrollo de habilidades lingüísticas que conduzcan a un buen orador, ya sea para un foro jurídico o un podio internacional.

A los efectos de la didáctica del derecho, son ineludibles no solo los contenidos asociados a la política interior, sino también a política exterior, estudiados por el derecho y las relaciones internacionales respectivamente. Como acertadamente sostiene Calduch (2018: 16):

La histórica separación que ha existido entre estas políticas ha sido paulatinamente diluida por la combinación de una progresiva mundialización de las relaciones y un constante desarrollo económico, tecnológico y comunicativo transnacional, cuestionando cada vez más el paradigma del Estado soberano y entronizando la constatación de una creciente interdependencia compleja que se impone a todos los actores internacionales. 
Tabla 1. Competencias específicas del grado de Relaciones Internacionales

CE01. Conocer los conceptos y técnicas aplicadas al análisis de los actores y relaciones internacionales.

CE02. Analizar la naturaleza y características de las relaciones entre los actores internacionales y particularmente entre los Estados.

CE03. Conocer y comprender los principales elementos que definen la política exterior de los Estados.

CE04. Relacionar los procesos y sucesos históricos con el orden internacional del presente.

CE05. Conocer y comprender tanto las teorías como las concepciones de las relaciones internacionales en perspectiva histórica.

CE06. Diferenciar las distintas competencias y funciones del Estado, las organizaciones internacionales y los actores no estatales en el sistema internacional.

CE07. Conocer las características de la geopolítica mundial y el concepto de la denominada sociedad internacional.

CE08. Conocer y comprender la situación política, social, económica y geoestratégica de cada una de las regiones a nivel internacional (Asia, América, África y Europa).

CE09. Comprender la estructura, las competencias y el funcionamiento tanto de las instituciones como de las organizaciones internacionales.

CE10. Comprender las instituciones, normas, procedimientos de decisión y políticas de la Unión Europea.

CE11. Comprender los fundamentos teóricos generales de la economía y de la estructura económica internacional.

CE12. Identificar y comprender tanto los datos macroeconómicos como los principales flujos económicos para analizar la coyuntura económica mundial.

CE13 - Conocer y analizar las teorías e ideologías políticas contemporáneas.

Fuente: Universidad de Navarra, España.

Luego de la referencia a destrezas y habilidades en sentido lato, es menester incurrir en el terreno de lo que metodológicamente contribuiría a diseñar la estrategia pedagógica, planteando la funcionalidad de los currículos diseñados por competencias. Siguiendo esta línea, en primer lugar se han analizado dos planes de estudios de relaciones internacionales: ${ }^{2}$ el de la Universidad de Navarra en España (tabla 1) y el del ISRI de Cuba (tabla 2), de manera que se extraigan las principales competencias que ameritan su incentivo la aprehensión de esta disciplina en el ámbito del derecho.

A tono con lo anterior, aparecen algunas invariantes del conocimiento a incorporar en el abordaje de la didáctica de las relaciones internacionales, de manera que promuevan el pensamiento crítico del estudiante de derecho. Dichas invariantes son

2. La selección de estos dos planes de estudios se debe a criterios científico-contextuales, toda vez que el caso de Navarra se incluye teniendo en cuenta la influencia de la Escuela Española de Relaciones Internacionales, una de las más antiguas y reconocidas en todo el orbe, con referentes teóricos brindados por destacados internacionalistas como Truyol, Del Arenal y Calduch. Se contrasta además el currículo del ISRI, por constituir el único instituto en el territorio nacional dedicado a la especialidad de las Relaciones Internacionales, lo cual conlleva a validar con mayor precisión la estrategia propuesta para el caso cubano. 
Tabla 2. Competencias profesionales del egresado de la carrera de Relaciones Internacionales en Cuba

\footnotetext{
Tener un sólido dominio de la realidad nacional y de las características, principios y prioridades estratégicas, políticas, jurídicas, económicas y de defensa de la Revolución cubana.

Valorar e interpretar una situación internacional dada, sus raíces históricas y sus consecuencias políticas, económicas y mediáticas, en especial para Cuba.

Hacer pronósticos y proponer cursos de acción útiles tanto para el diseño como para la ejecución de la política exterior de la Revolución cubana. También para el desarrollo de sus nexos en el ámbito internacional.

Mostrar, en todo momento, una actitud que se corresponda con los principios y valores de la Revolución, las tradiciones patrióticas de nuestro pueblo, el legado de nuestros mártires, el sentir solidario e internacionalista, y una concepción científica marxista leninista de la realidad política, económica y social en los diferentes escenarios a partir de las posiciones de la Revolución cubana.

Fuente: Plan de estudio de Relaciones Internacionales.
}

política exterior, actores internacionales y coyuntura económica mundial, las cuales constituyen una síntesis de las competencias específicas de los planes de estudios de relaciones internacionales de la Universidad de Navarra y del ISRI. En tanto la política exterior se configura como un ente complejo, los discursos, conferencias y otras fuentes documentales informan al estudiante de los pilares fundamentales que enarbola. También, mediante los Modelos de Naciones Unidas (MNU), se propicia la adquisición de conocimientos trascendentes a los asuntos exteriores de cualquier Estado y, en particular, se desarrolla el diálogo socrático aplicado al ámbito educativo, según Del Mastro (2020).

Con los MNU se realiza una simulación del actuar del funcionamiento de las Naciones Unidas, específicamente en el desenvolvimiento de las relaciones políticas a través de la representación por comisiones de sesiones de la Asamblea General, el Consejo de Seguridad, y otros órganos, así como organizaciones afines. El dominio de las posturas oficiales de los Estados miembros retroalimenta - en esta simulación- los discursos preparados por los delegados, donde se fomentan otras competencias encaminadas a conocer las alianzas, bloques regionales y la cooperación internacional. Esto permite desarrollar el método interactivo, que promueve dinámicamente la relación entre estudiantes y el vínculo estudiantil con el claustro en su función evaluadora.

Con respecto a los actores internacionales, lo que pretende es desmitificar el hecho de que el Estado-Nación constituye el protagonista en las relaciones internacionales en la actualidad, y esta postura es la que visualizan los estudiantes de derecho, toda vez que desde derecho internacional público los Estados constituyen el principal sujeto de derecho internacional; y en derecho internacional privado, si bien el Estado no es el eje central de las relaciones en cuestión, pueden serlo, aunque no en base a la soberanía ejercida en el ámbito público. Se pretende entonces instar a los estudios de otros actores internacionales como la prensa internacional. 
De hecho, se puede constatar que la prensa internacional constituye un elemento trascendental para visualizar el cumplimiento de los derechos humanos a nivel internacional. El factor mediático es situado dentro de los factores intelectuales del sistema internacional. Como alude el propio Rodríguez (2017: 135), la mediatización de la vida internacional produce efectos variables sobre diferentes situaciones, entre los que se encuentran las presiones a favor del respeto de los derechos humanos según los cánones occidentales.

Teniendo en cuenta lo anterior, la asunción de la opinión pública internacional resulta vital en los estudios interdisciplinares, toda vez que cada rama del derecho está asociada al cumplimiento de los derechos humanos. El estudiante de derecho debe conocer los principales debates ocasionados en los grandes medios de prensa. Si bien existe también un influjo de información a través de medios informales en la sociedad virtual, el estudiante debe contrastar las realidades difundidas y aprovechar oportunidades para la argumentación jurídica en los medios de prensa oficial.

La contextualización de los contenidos de aprendizaje supera el uso tradicional de materiales impresos y permite conectar - digitalmente-conceptos teóricos con el mundo real, para lo que el estudio de la coyuntura suele ser un potente motivador para los estudiantes durante el proceso de enseñanza. Tanto es así que un estudio realizado en Uruguay propone la introducción del análisis de artículos de prensa, tapas de revistas como The Economist, Time o The New Yorker, o de caricaturas que ilustraban diferentes aspectos del comercio internacional o del impacto de la covid-19 en las relaciones económicas mundiales y la gobernanza global (Bas, 2020: 6).

En aras de contribuir con la formación integral del jurista, la adaptación de una estrategia para el estudio de las relaciones internacionales en la carrera de Derecho debe fomentarse interdisciplinariamente a partir de las competencias específicas. La conexión académica de estas dos ciencias no pretende desvirtuar la esencia de lo que importa a cada una, ni pretende exigirle a un jurista llegar a ser un analista internacional, sino permitirle una mayor aprehensión del sistema internacional para solucionar problemáticas generales del derecho.

\section{Estrategia para la formación en relaciones internacionales en la carrera de Derecho en Cuba}

Según Delgado, «los estudios de derecho en Cuba son coetáneos a los inicios de la enseñanza universitaria en el país» (Delgado, 2017: 1), por lo que se aduce que datan de 1728, cuando se fundó la Real y Pontificia Universidad de San Jerónimo de La Habana. Por ende, a más de tres siglos de enseñanza del derecho en la isla, el perfeccionamiento de los procesos universitarios clama por una nueva epistemología científica y pedagógica en la educación superior. De hecho, estudios recientes desde la ciencia cubana validan la necesidad de perspectivas integradoras en los procesos 
de enseñanza aprendizaje. Es por esto que el catedrático Núñez (2020: 5) hace alusión a Richard Levins, en el sentido de que este «se quejaba de la supervivencia de los enfoques simplificados que impiden lidiar con la complejidad y la superespecialización que limita la integración».

El estudio pedagógico en cuestión lleva, en primera instancia, a identificar qué contenidos de las relaciones internacionales están vigentes en el plan de estudios de la carrera de Derecho en Cuba. Autores como Páez, Silva y Trujillo (2020: 293) han abordado el tema, aludiendo a que el proceso de formación de juristas para las relaciones internacionales consiste en:

La sucesión de etapas en las que se prepara integralmente al estudiante de derecho para argumentar el desarrollo de las relaciones multifacéticas internacionales con sustento en los principios, normas e instituciones del derecho internacional en los ámbitos público y privado, desde las dimensiones curricular y extracurricular.

Con respecto a la disciplina de derecho internacional, es necesario definir el proceso formativo que en torno a ella se debate en el ámbito jurídico. No obstante, según Velázquez (2006: 831):

En la actual sociedad globalizada, el derecho internacional ha dejado ya de ser materia exclusiva de las escuelas y facultades de Derecho para convertirse en un patrimonio disciplinario compartido con las ciencias políticas y sociales, particularmente con la ciencia de las relaciones internacionales.

Teniendo en cuenta la importancia del derecho internacional en las relaciones internacionales, primero se analiza la situación actual de la asignatura en la carrera de Derecho en Cuba. Por su parte, en el actual plan de estudios, el objeto de la disciplina científica del derecho internacional lo constituyen «las relaciones multifacéticas de carácter internacional, tanto públicas como privadas». Esta disciplina desempeña un papel relevante en la formación del jurista, resultante del proceso de internacionalización de la vida. Habida cuenta, actualmente no es posible concebir una rama del derecho desvinculada de la vis internacional, ya sea por la presencia del elemento extranjero en la relación jurídica que se analice, como por la presencia de normas, instituciones y figuras del derecho internacional que inciden de manera directa en la vida jurídica de un país.

Lo que sucede en la enseñanza de las relaciones internacionales en la carrera de Derecho en Cuba, es que el manejo de conceptos recae subrepticiamente en una parcela de la realidad internacional. A esto, se suma que la complementariedad de estudios no se logra en la formación posgraduada, ya que los egresados de derecho no suelen optar por los programas académicos ofertados en La Habana, como la maestría en Relaciones Internacionales. 
Con respecto a la asignatura de Derecho internacional público, el término relaciones multifacéticas suele confundirse con el de relaciones internacionales, aun cuando este último adquiere fenomenología como ciencia. Por lo tanto, suele sostenerse la creencia errónea de que el derecho internacional abarca el sistema internacional y no es menos cierto que es parte fundamental de él, pero lo trasciende. Por ende, la dimensión jurídica no es la única a tener en cuenta, ya que las relaciones internacionales se extienden más allá, a los escenarios de la diplomacia, el protocolo internacional, la política exterior, la política internacional y hacia otras disciplinas distintas al derecho internacional.

En este punto de análisis, surge la necesidad de una estrategia, identificada como aporte investigativo, en este caso, al tratarse de una estrategia pedagógica definida por Valle (2007: 94) como «el conjunto de acciones secuenciales e interrelacionadas que, partiendo de un estado inicial y considerando los objetivos propuestos, permite dirigir y organizar de forma consciente e intencionada (escolarizada o no) la formación integral de las nuevas generaciones».

Para transformar el modelo actuante, la estructura de la estrategia diseñada incluiría la realización de un diagnóstico previo a través de una matriz DAFO, el diseño de un objetivo general y la fundamentación de acciones estratégicas específicas. En este sentido, se prevería tanto el empleo de métodos y procedimientos, así como la disponibilidad de los recursos materiales y humanos necesarios para lograr su materialización.

El diagnóstico inicial incluyó el empleo de métodos como la observación científica y las entrevistas grupales al colectivo de la disciplina: derecho internacional, integrado por cuatro profesores en la Universidad de Pinar del Río. Con los resultados de aplicación de estos métodos empíricos, se fue construyendo la matriz DAFO, que en el orden interno contiene fortalezas y debilidades, y en el plano externo incluye amenazas y oportunidades.

Las fortalezas identificadas resultaron ser:

- El adecuado diseño curricular de la disciplina de derecho internacional en el plan de estudios vigente para todo el territorio nacional, cuya estructuración incluye dos asignaturas: derecho internacional público y derecho internacional privado.

- El estudiantado de la carrera de Derecho muestra motivación por participar anualmente como delegados en los Modelos de Naciones Unidas.

- El claustro jurídico reconoce la importancia de la formación para las relaciones internacionales en el ámbito jurídico. 
- La existencia de siete Modelos de Naciones Unidas en el país. ${ }^{3}$

- Las debilidades fueron:

- Insuficiente especialización del claustro de derecho en materia de relaciones internacionales.

- Deficiente transversalidad del estudio de las relaciones internacionales en el currículo de la carrera de Derecho.

- Insuficiente desarrollo de investigaciones del colectivo pedagógico en la materia.

- Escasa socialización de resultados científicos del claustro en revistas de alta visibilidad en materia de relaciones internacionales.

- Inexistencia de Modelos de Naciones Unidas en ocho provincias de la Isla de Cuba.

Las amenazas resultaron ser:

- La insuficiente concepción de figuras de posgrado (maestrías, especialidades y doctorados) en Cuba que tributen a la especialización en materia de relaciones internacionales.

- La tendencia al ejercicio de la diplomacia en estudiantes universitarios residentes de la capital cubana, y su menor frecuencia en el resto de las provincias.

Las oportunidades reflejaron:

- La existencia, en todas las provincias, de Capítulos Derecho Internacional de la Unión Nacional de Juristas de Cuba, encargados del desarrollo de sesiones científicas en la materia.

- La vinculación universitaria con otras instituciones y organismos del territorio nacional, como el Instituto Cubano de Amistad con los Pueblos, el Ministerio del Interior, la Cruz Roja, entre otros.

- La existencia de un Instituto Superior de Relaciones Internacionales en Cuba para el desarrollo de la cooperación científica y el establecimiento de vínculos tanto académicos como culturales con otras universitarias patrias y del orbe.

3. Los Modelos de Naciones Unidas en Cuba se circunscriben al ámbito universitario, uno por provincia, salvo en el caso de la capital cubana, que tiene dos modelos. La representatividad territorial de Occidente a Oriente es la siguiente: ONUPINAR en Pinar del Río, AGORA y HAVMUN en La Habana, PUENTES en Matanzas, ORBIS en Villa Clara, ONUCARIBE en Santiago de Cuba y CARAPACHIBEI en la Isla de la Juventud. 
- La oferta de posgrado de la maestría en Relaciones Internaciones convocada por el ISRI.

Una vez identificadas las potencialidades, barreras, limitaciones del objeto de estudio y sus manifestaciones, teniendo como punto de referencia la Universidad de Pinar del Río, se asume que la propuesta es generalizable a todo el país, habida cuenta de que el plan de estudios $\mathrm{E}$ es de alcance nacional y abarca a todas las universidades del territorio cubano. Por ende, como objetivo general de la estrategia se prevé: perfeccionar el proceso formativo para las relaciones internacionales en la carrera de Derecho en Cuba, con carácter sistémico e integrado. Para estos fines, se han diseñado tres acciones estratégicas específicas:

Rediseño didáctico de la disciplina del derecho internacional.

Transversalidad de las relaciones internacionales en el currículo jurídico.

Concepción de un sistema de actividades extracurriculares para la formación en relaciones internacionales.

Por lo tanto, el rediseño didáctico es la primera acción estratégica específica, orientada a perfeccionar el currículo jurídico a tono con el desarrollo actual de las relaciones multifacéticas internacionales. En primer lugar, es válido destacar que el diseño curricular actual de la disciplina derecho internacional amerita ser revisado, teniendo en cuenta el examen de sus componentes didácticos. Un elemento esencial resulta ser el sistema de habilidades existente, toda vez que la tendencia contemporánea sugiere el diseño curricular por competencias, tal cual los diseños supra aludidos de la Universidad de Navarra en España y del Instituto Superior de Relaciones Internacionales en Cuba. Este es precisamente uno de los principales retos de la formación jurídica para las relaciones internacionales, que requiere la previsión de competencias comunicativas (lingüísticas, oratorias, de idiomas y de protocolo), competencias jurídicas genéricas (en el ámbito de las relaciones internacionales) y competencias argumentativas específicas (retóricas y litigantes para la solución de conflictos suscitados en la comunidad internacional).

Otro aspecto a tener en cuenta en el rediseño didáctico es el reducido espectro de la disciplina a solo dos asignaturas (Derecho internacional público y privado). En ese sentido, se advierte la necesaria previsión de materias optativas, como derecho internacional humanitario, derecho internacional de los derechos humanos, derecho de tratados, derecho de extranjería y derecho procesal internacional, las cuales no se han aprovechado al máximo en la configuración del plan del proceso docente en todas las universidades del país.

No obstante, la diversificación o ampliación de asignaturas no sería suficiente ni exclusiva para la solución del problema pedagógico en cuestión, sino que, desde las materias ya existentes, se puede lograr el pretendido empeño si se logran adecuadas adaptaciones curriculares, según Ridao (2016). 
Asignaturas como Litigación, impartida en la Universidad de Pinar del Río, ameritan incorporar estudios de conflictos internacionales a partir de la teoría de relaciones internacionales, lo cual sería un enclave fundamental en el apoderamiento de nuevos contenidos. De esta forma, empleando criterios doctrinales como los del autor cubano Rodríguez, el estudiante puede estudiar conflictos de larga data a través del análisis de los elementos del sistema internacional.

En ese sentido, Silva y Carballo (2019) valoran el conflicto suscitado en la península coreana, teniendo en cuenta el desarrollo de las relaciones internacionales como componente esencial del Derecho Internacional Público, con énfasis en el comportamiento del equilibrio de fuerzas, el factor militar y el factor mediático, lo cual podría aportar un análisis políticamente vital en los estudios de la asignatura Derecho internacional.

Precisamente, la segunda acción estratégica alude a la transversalidad, en tanto esta opera como eje dinamizador de los contenidos de internacionalización en toda la carrera. Tanto es así que, desde el resto de las disciplinas científicas y años académicos, se puede potenciar la formación del estudiante de derecho para las relaciones internacionales. De hecho, un elemento interesante del actual plan de estudios resulta la configuración de estrategias transversales al macro, meso y micro currículo. Entre ellas, se encuentran la estrategia para la comunicación en idioma extranjero, la económica, la de preparación para la defensa, la estrategia de preparación políticoideológica, la medioambiental y la histórica. Todas estas tributan a la articulación de conocimientos, habilidades y valores en la carrera de Derecho.

Por su parte, las disciplinas científicas del derecho evidencian potencialidades para diversificar la formación internacionalista del estudiante. Así las cosas, desde la disciplina fundamentos teóricos e históricos del Estado y el derecho, se estudian contenidos referidos al diferendo histórico Cuba-Estados Unidos, la vigente Ley-Helms Burton, la situación jurídica de la Base Naval de Guantánamo y el bloqueo económico, financiero y comercial impuesto a Cuba como medida coercitiva unilateral. Asimismo, se estudian entes del derecho público, contratos y convenios internacionales, resoluciones del Consejo de Seguridad de las Naciones Unidas, y las principales corrientes políticas y dogmáticas que tratan de explicar el actual sistema de relaciones internacionales, cuestiones asociadas a las inmunidades soberanas de los Estados, el mantenimiento de la paz y la seguridad internacional.

Desde la disciplina del derecho de la empresa, se estudia lo relativo al arbitraje comercial internacional, se analiza el orden económico internacional, las particularidades de la inversión extranjera y los denominados Acuerdos de Promoción y Protección Recíproca de Inversiones. De igual manera, en el derecho ambiental se pormenoriza en la tarea vida, llevada a cabo por el Estado cubano para el enfrentamiento al cambio climático y la preservación del medio ambiente sano, a partir de la firma de tratados internacionales en la materia. 
Las relaciones transdisciplinares en la carrera enfocadas a la disciplina del derecho penal y criminología, se visualizan desde el estudio de instituciones jurídicas como la extradición y el funcionamiento de institutos internacionales como la Corte Internacional de Justicia y la Corte Penal Internacional. La disciplina del derecho civil y familia tiene un fuerte componente teórico, como precedente del derecho internacional privado por la supletoriedad del derecho civil y desde el derecho notarial en lo referido a la actuación consular.

En tercera instancia, como ciertamente apunta Velázquez (2006: 845), «la lección catedrática del derecho internacional debe ser trascendida», de manera que la formación para las relaciones internacionales debe superar la enseñanza tradicional y asumir métodos creativos para superar «los riesgos en la tradición formalista de enseñanza del derecho» (Ramallo, 2020: 10). En este empeño, también ocupa un lugar primordial el empleo del método interactivo y de métodos problemáticos, como los juegos de roles. En particular, en Cuba, se ha diseñado una variante extracurricular que alude a los Modelos de Naciones Unidas, ejercicios de simulación que brindan la posibilidad a los cuasi juristas de fungir como representantes de determinados países y defender sus posturas oficiales ante la-simulada-comunidad-internacional. Estas experiencias pedagógicas de los concursos Moot, son prácticas asumidas por estudiantes de numerosos centros de educación superior en el mundo a criterio de Ramallo (2020).

De hecho, el Plan E de estudios privilegia aún más el autoaprendizaje del estudiante. En consecuencia, la actividad docente-metodológica habrá de encaminarse necesariamente al diseño de métodos que permitan la orientación y el control del proceso docente con una concepción pedagógica que propenda a activar el papel del alumno como protagonista de su propia formación (Delgado, 2017: 7). Así, los juegos de roles aparecen como estrategias pedagógicas válidas para el aprendizaje activo $\mathrm{y}$ la enseñanza problemática, siendo esencial el desarrollo de la controversia en tanto «algunas escuelas han incorporado los «temas controversiales» en el currículo como una estrategia para promover la futura participación política de sus estudiantes» (Magendzo y Toledo, 2015: 13).

Ante este reto del nuevo plan de estudios de la carrera de Derecho en Cuba, los procesos extensionistas ocupan un lugar importante en la autopreparación del estudiante, específicamente en torno a las relaciones internacionales. La citada experiencia cubana en los Modelos de Naciones Unidas ha demostrado una dimensión desarrolladora de habilidades y valores en los estudiantes, que fungen como delegados, oyentes e incluso Comité Organizador de los eventos que derivan de los MNU.

Por otra parte, la enseñanza del derecho internacional público puede potenciarse desde el estudio de la jurisprudencia internacional, para lo cual puede resultar útil el empleo del método de casos. Esta sería una variante pedagógica para acercar al discente a la praxis internacional en los ámbitos judicial, arbitral, político o diplomático. 
De manera particular, resalta la aproximación a la práctica jurídica internacional, al analizar tanto laudos arbitrales como casos reales, fallos judiciales y precedentes sentados por instancias jurisdiccionales como la Corte Internacional de Justicia, la Corte Penal Internacional y los Tribunales Penales ad hoc (para la ex Yugoslavia y para Ruanda respectivamente), así como las diversas cortes de derechos humanos.

A grandes rasgos, las tres acciones estratégicas dirigidas a perfeccionar el proceso formativo del jurista para las relaciones internacionales, desde su componente curricular y extracurricular, permiten vislumbrar un redimensionamiento útil para juristas y didactas desde una visión inter, multi y transdiciplinar. Es por esto que Estrada y Reyes (2015: 913) afirman razonadamente que:

En la pedagogía moderna del derecho, debe tenerse bien claro el significado y relevancia de trabajar coordinada y conjuntamente con otros científicos y especialistas afines, para posibilitar así el ideal cognoscitivo de la interdisciplinariedad y la integración del conocimiento.

La estrategia cuenta con tres etapas, secuenciadas a partir de un diagnóstico inicial que permitió identificar las fortalezas, debilidades, amenazas y oportunidades. La primera etapa (en curso) está dirigida al diseño de las tres acciones estratégicas específicas. La segunda etapa se corresponde con la implementación de la estrategia, que ha sido relegada en el contexto pandémico del 2020. Por último, se prevé la fase final de evaluación de la estrategia mediante métodos teóricos, empíricos y estadísticos, que permitan validar la trascendencia de la metodología propuesta y su impacto en la práctica pedagógica universitaria.

La implementación de la estrategia pedagógica permitirá determinar el nivel de conocimiento de los estudiantes al integrar contenidos iusinternacionales durante la carrera, el nivel de preparación de los profesores para transversalizar la materia en el currículo, y el grado de pertinencia de las acciones estratégicas implementadas desde lo curricular y extracurricular. De esta manera, durante las tres fases: diseño, implementación y validación de la estrategia, se logrará un proceso de retroalimentación que permita evaluar de manera objetiva su impacto en el proceso formativo estudiado.

\section{Conclusión}

La ciencia del derecho, enfocada en su objeto a las relaciones jurídicas, y la ciencia de las relaciones internacionales, abocada al estudio de la sociedad internacional, tienen múltiples puntos coincidentes y, a su vez, trascendentes al derecho internacional. En pos de su efectiva enseñanza, la didáctica del derecho debe enfocarse hacia los retos de la actual comunidad internacional, unipolar y globalizada, a la vez que debe asumir los enfoques pedagógicos orientados a una formación por competencias. 
Es una pretensión necesaria que el estudiante de derecho evalúe adecuadamente las normas, instituciones y doctrinas del derecho internacional contemporáneo, pero, a su vez, se requiere que este realice estudios internacionales que trasciendan el iusinternacionalismo. Esta perspectiva holística coadyuvaría a perfeccionar el proceso de enseñanza-aprendizaje para las relaciones internacionales, direccionando la formación integral del jurista en el pregrado universitario.

La propuesta de una estrategia pedagógica para la enseñanza de las relaciones internacionales en Cuba es pertinente, novedosa y viable para las universidades del territorio nacional, en tanto concibe tres acciones estratégicas específicas, orientadas al rediseño didáctico de la disciplina del derecho internacional, al logro de la transversalidad del estudio de las relaciones internacionales en el currículo jurídico y a la implementación de un sistema de actividades extracurriculares en materia internacional para la formación integral del jurista. Su impacto se medirá a mediano plazo cuando se completen las tres etapas de diseño, implementación y validación, toda vez que hasta la fecha solo se ha consumado la fase inicial, susceptible de perfeccionamiento mediante actividades como las modelaciones de Naciones Unidas y otras estrategias pedagógicas que impacten en el currículo jurídico.

\section{Referencias}

Álvarez, Carlos Manuel (1999). Didáctica. La escuela en la vida. La Habana: Pueblo y Educación.

Bellver, Vicente (2019). «Educar en derechos humanos: Orientaciones del derecho internacional e implementación en la educación superior». Revista de Educación $y$ Derecho, 20: 1-25. Disponible en bit.ly/3nDLhKI.

BAS, Magdalena (2020). «Enseñar relaciones internacionales en tiempos de covid-19: Desafíos didácticos desde la enseñanza virtual». Fundación Carolina, 35: 1-12. Disponible en bit.ly/2Ja3Bw1.

CALDUCH, Rafael (2011). Relaciones internacionales. Madrid: Ciencias Sociales.

Calduch, Rafael (2013). «La Escuela española de Relaciones Internacionales». Revista de Relaciones Internacionales de la UNAM, 115: 9-32. Disponible en bit. ly/2JaFAVN.

-. (2018). «La transición entre sociedades internacionales y el derecho internacional público». Anuario Español de Derecho Internacional, 34: 29-50. Disponible en bit. ly/2Khjxxi.

Delgado, Teresa (2017). «La enseñanza del derecho en Cuba». Florida journal of international law, 29: 1-7. Disponible en bit.ly/2WCUBTn.

Del Mastro, Fernando (2020). «El ethos socrático y la formación del ser en la enseñanza del derecho». Revista Pedagogía Universitaria y Didáctica del Derecho, 7 (1), 195-224. Disponible en bit.ly/38vM6PE. 
EstradA, Guillermo y Carlos Reyes (2015): «La enseñanza del derecho y del derecho internacional». Anuario Mexicano de Derecho Internacional, 15: 909-913. Disponible en bit.ly/2KhToOV.

FARFÁn, Enrique (2019). «La visión integral de la formación jurídica que surge de la retórica». Revista Pedagogía Universitaria y Didáctica del Derecho, 6 (2): 5-20. Disponible en bit.ly/3rhIcCq.

García, José Antonio (2017). «El giro del derecho internacional a las relaciones internacionalesen Hans J. Morgenthau: Una transición mediada por la historia». Grupo de Estudios de Relaciones Internacionales (GERI), 37: 31-57. Disponible en bit.ly/38tKO7u.

Hernández, Diana (2018). «Estrategias pedagógicas para una mejor enseñanza de estudios superiores en derecho a estudiantes mayores». REJIE: Revista Jurídica de Investigación e Innovación Educativa, 18: 57-79. Disponible en bit.ly/3mFoNaM.

IzQuiERDO, María José (2019). «10o años de estudios de Relaciones internacionales». Documento informativo IEEE, 12/2019. Disponible en bit.ly/3nFyPdy.

Magendzo Kolstrein, Abraham y María Isabel Toledo Jofré (2015). «Educación en derechos humanos: Estrategia pedagógica-didáctica centrada en la controversia». Revista Electrónica Educare, 19( 3): 16. Disponible en bit.ly/3pdiBZn.

Narváez, José Ramón (2019). «Metodología crítica para la investigación científica del derecho». Revista Pedagogía Universitaria y Didáctica del Derecho, 6 (2): 81-95. Disponible en bit.ly/3rjTy8L.

NúñEZ, Jorge (2020). «Pensar la ciencia en tiempos de covid-19». Revista Anales de la Academia de Ciencias de Cuba, 10: 1-6. Disponible en bit.ly/3rdZdxr.

PÁez, Lisett D., Jorge Luis Silva y Mercedes Trujillo (2020). «Retos de la formación de juristas para las relaciones internacionales en Cuba». Revista Conrado, 16 (74): 291-297. Disponible en bit.ly/38vNsKe.

Prieto, Martha (2019). El derecho como ciencia. Disponible en bit.ly/2J803KG.

RODRíGUEZ, Leyde Ernesto (2017). Un siglo de teoría de las relaciones internacionales. La Habana: Félix Varela.

Ramallo, María de los Ángeles (2020). «La tradición formalista en la enseñanza del derecho y su impacto en la formación práctica: Estudio de caso del patrocinio jurídico de la Universidad de Buenos Aires». Revista Pedagogía Universitaria y Didáctica del Derecho, 7 (1), 9-41. Disponible en bit.ly/3nH9pfp.

RIDAO, Joan (2016). «La enseñanza del derecho constitucional mediante las TIC. Un estudio de caso no-presencial». REJIE: Revista Jurídica de Investigación e Innovación Educativa, 13: 49-62. Disponible en bit.ly/2WAfQoP.

SALvioli, Fabián (2002). «Algunas consideraciones sobre la enseñanza contemporánea del derecho internacional público». Revista de Relaciones Internacionales, 22: 1-20. Disponible en bit.ly/3mDiyOL. 
SiLVA, Jorge Luis y Adoración Carballo (2019). «La persistencia del conflicto en la península coreana: Reflexiones desde el derecho internacional público». Revista Márgenes, 7 (2): 60-78. Disponible en bit.ly/34tYdLN.

Sipaco, Narciso (2014). El laboratorio de las relaciones internacionales públicas y diplomáticas. Pinar del Río: Hermanos Loynaz.

SolAnES, Ángeles (2018). «La importancia de la filosofía del derecho en los planes de estudios jurídicos dentro del EEES». Revista Educación y Derecho, 17: 2-23. Disponible en bit.ly/2J7YFHY.

Truyol, Antonio (1973). La teoría de las relaciones internacionales como sociología (Introducción al estudio de las relaciones internacionales). Madrid: Instituto de Estudios Políticos.

Universidad de Valladolid (2015). Guía docente de la asignatura. DisponiBLE EN BIT.LY/3PBKDOB.

VALLE, Alberto (2007). Algunos modelos importantes en la investigación pedagógica. La Habana: Instituto Central de Ciencias Pedagógicas.

Villalpando, Waldo (2009) «La formación del jurista objetivos y competencias en la enseñanza del derecho». Revista Invenio, 12 (22): 13-28. Disponible en: bit. ly/3nGGVT5.

VelázQuez, Juan Carlos (2006). «Orientaciones básicas acerca de la enseñanza del derecho internacional en ciencias políticas y sociales». Anuario Mexicano de Derecho Internacional, 6: 831-869. Disponible en bit.ly/3mEt52n.

\section{Reconocimiento}

Esta investigación es resultado del Proyecto de Innovación «Modelo de formación integral del profesional del Derecho» (PI-145). Actualmente, se encuentra en ejecución en la Universidad de Pinar del Río de Cuba (2017-2020).

\section{Sobre las autoras}

Adoración Carballo Moya es maestrante en la XII Edición de la Maestría en Ciencias de la Educación del Centro de Estudios de Educación Superior de la Universidad de Pinar del Río, Cuba. Se graduó como licenciada en Derecho con Premio al Mérito Científico en la Universidad de Pinar del Río, Cuba. En 2018, obtuvo el Premio Nacional «Miguel D’ Estéfano Pisani» en Derecho Internacional, otorgado por la Unión Nacional de Juristas de Cuba. Además, es miembro del Capítulo Provincial de la Sociedad Cubana de Derecho Internacional. Ha publicado artículos científicos en revistas especializadas como Márgenes (Cuba) y Cuaderno de Pedagogía Universitaria (República Dominicana). Su correo electrónico es adoracioncarballomoya@gmail. com. (D) https://orcid.org/0000-0002-6738-5705. 
Lisett D. PÁez Cuba es doctora en Ciencias Pedagógicas y Máster en Ciencias de la Educación. Se graduó como licenciada en Derecho con Título de Oro en la Universidad de Pinar del Río, Cuba. En 2017, obtuvo el Premio Nacional «Joven investigador» en Ciencias Pedagógicas, otorgado por el Ministerio de Ciencia, Tecnología y Medio Ambiente en Cuba. Ha realizado estancias de investigación en la Universidad de Granada, España, y en la Universidad de Estocolmo, Suecia. Ha publicado artículos científicos en revistas especializadas como Pedagogía Universitaria (Cuba), Oralia. Análisis del Discurso Oral (España), Revista de Educación y Derecho (España), y Cuaderno de Pedagogía Universitaria (República Dominicana). Es coordinadora del proyecto de innovación «Modelo de formación integral del profesional del Derecho» (PI-145), en ejecución durante el período 2017-2020. Es vicepresidenta del Capítulo Provincial de la Sociedad Cubana de Derecho Internacional. Actualmente, funge como vicedecana de Investigación y Posgrado de la Facultad de Ciencias Sociales y Humanidades de la Universidad de Pinar del Río, Cuba. Su correo electrónico es lisett@upr.edu.cu. (D) https://orcid.org/o0oo-0001-8907-7562. 